\title{
Cryo-EM Structure of the Single-Ring Chaperonin from Bacteriophage OBP $P$. fluorescence
}

\author{
T.B. Stanishneva-Konovalova ${ }^{1}$, P.I. Semenyuk ${ }^{2}$, Y. Pechnikova ${ }^{3}$, L.P. Kurochkina ${ }^{4}$ and O.S. Sokolova ${ }^{1}$ \\ 1. M.V. Lomonosov Moscow State University, Faculty of Biology, Moscow, Russia. \\ 2. M.V. Lomonosov Moscow State University, A.N. Belozersky Institute of Physical-Chemical Biology, \\ Moscow, Russia. \\ 3. Materials \& Structural Analysis, Thermo Fisher Scientific, Eindhoven, Netherlands. \\ 4. Shemyakin-Ovchinnikov Institute of Bioorganic Chemistry, Russian Academy of Sciences, Moscow, \\ Russia
}

Chaperonins are the Hsp60 (heat-shock protein 60) family of molecular chaperones, which ensure the correct folding of denatured or newly synthesized proteins. They consist of several subunits organized in barrel-like structures with an inner cavity where the folding occurs. The common feature of all chaperonins is the ATP-dependent transition between open and closed conformations. Recently, chaperonins have been found in bacteriophage genomes [1,2]. The object of the present study is a single ring chaperonin of bacteriophage OBP $P$. fluorescence.

The 3D structure of OBP chaperonin was studied here by cryo-electron microscopy. The protein was isolated from E. coli lysate and purified using Q sepharose chromatography. Data collection was performed at NeCen (Leiden, Netherlands). Over 2000 images were recorded on the Titan Krios microscope equipped with a Falcon 2 direct detector using magnification that resulted in a pixel size of $0.78 \AA$. The automatic particle selection was accomplished in Relion2.1 [3]. After particle sorting, classification and visual inspection, 43098 particles were left for further processing. The refinement of the $3 \mathrm{D}$ reconstruction was done without imposing a symmetry. After the post-processing, the final resolution of the structure was estimated to be $7.3 \AA$, based on the gold-standard Fourier shell correlation at 0.143 .

The resulting structure of a bacteriophage encoded chaperonin OBP is a ring of seven subunits (Fig. 1A, B). This distinguishes it from most chaperonins, usually consisting of two rings. Despite being assembled from seven chemically identical subunits, it only displays C7 symmetry at the level of equatorial domains (Fig. 1C), while apical domains are arranged as three pairs and one unpaired domain (Fig. 1D). The unpaired subunit is less resolved in the reconstruction, which implies its higher mobility compared to subunits within pairs. This raises a question of whether the role of a 'free' subunit is played by the same subunit, or the roles may be switched. Cryo-EM data alone cannot answer this question since it only provides a static view. One possible approach to address this problem is to use computational simulations.

Fitting of a homology-based atomic structure into the density map indicated a novel subunit conformation: compared to GroEL, the apical domains of OBP chaperonin are rotated relative to the equatorial domains (Fig. 2). There is also a kink in the intermediate domain (Fig 2B). Structural differences between the OBP chaperonin and chaperonins of group I and group II indicate the existence of a yet unknown mechanism of chaperonin activity [4]. 
References:

[1] L.P. Kurochkina et al. J.Virol. 86 (2012), p. 10103.

[2] P.I. Semenyuk et al. Biochem J. 473 (2016), p. 2383.

[3] D. Kimanius et al. Elife 5 (2016), pii: e18722.

[4] The authors acknowledge funding from the Russian Foundation for Basic Research (Grant \#16-0401587 to O.S.S., \# to L.P.K.).
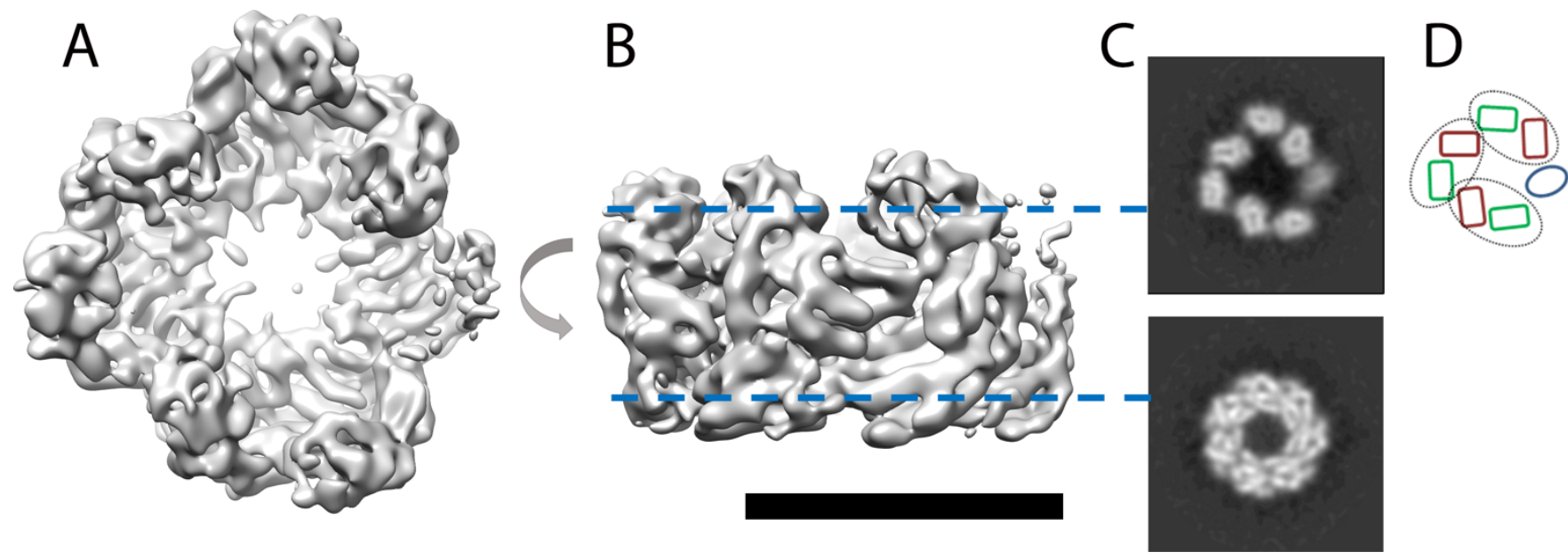

Figure 1. Cryo-EM structure of single-ring OBP chaperonin. (A) Symmetry-free 3D reconstruction (top view); (B) side view. Scale bar is $10 \mathrm{~nm}$. (C) sections through reconstructions at the level of apical domains (upper image) and equatorial domains (bottom image); (B) schematic, illustrating the hypothetical arrangement of the apical domains.
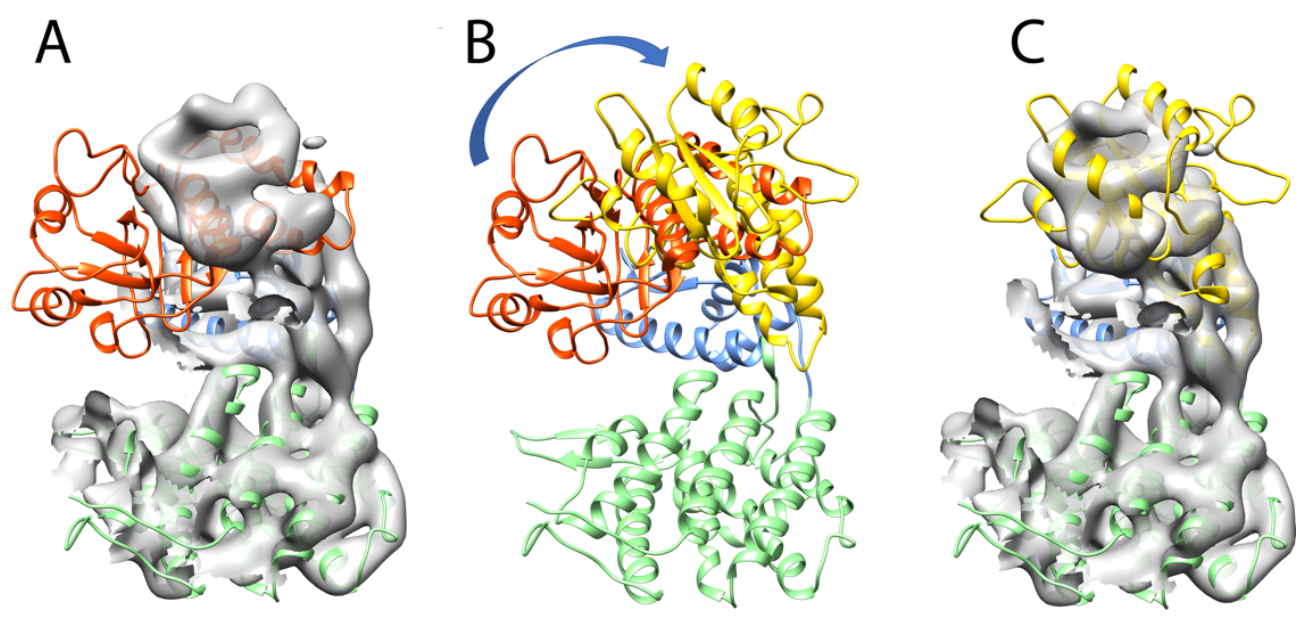

Figure 2. Fitting of the homology model of OBP chaperonin into EM density of a single subunit. (A) rigid fitting demonstrates inconsistence in the apical domain position; (B) conformational change, that includes the kink in the intermediate domain; (C) flexible fitting demonstrates good correlation. 